\title{
Effect of diastolic dysfunction on postoperative outcomes after cardiovascular surgery: A systematic review and meta-analysis
}

\author{
Roop Kaw, MD, ${ }^{\text {a,b }}$ Adrian V. Hernandez, MD, PhD, ${ }^{\text {,d }}$ Vinay Pasupuleti, MD, PhD, \\ Abhishek Deshpande, MD, PhD, ${ }^{\mathrm{f}}$ Vijaiganesh Nagarajan, MD, MRCP, ${ }^{\mathrm{g}}$ Hector Bueno, MD, PhD,,${ }^{\mathrm{h}, \mathrm{i} j}$ \\ Craig I. Coleman, PharmD, ${ }^{\mathrm{k}}$ John P. A. Ioannidis, MD, DSc, ${ }^{1}$ Deepak L. Bhatt, MD, MPH, ${ }^{\mathrm{m}}$ and \\ Eugene H. Blackstone, MD ${ }^{\mathrm{n}}$ for the Cardiovascular Meta-analyses Research Group
}

\begin{abstract}
Objective: The objective of this study was to investigate the effect of preoperative diastolic dysfunction on postoperative mortality and morbidity after cardiovascular surgery.
\end{abstract}

Methods: We systematically searched for articles that assessed the prognostic role of diastolic dysfunction on cardiovascular surgery in PubMed, Cochrane Library, Web of Science, Embase, and Scopus until February 2016. Twelve studies $(\mathrm{n}=8224)$ met our inclusion criteria. Because of the scarcity of outcome events, fixed-effects meta-analysis was performed via the Mantel-Haenszel method.

Results: Preoperative diagnosis of diastolic dysfunction was associated with greater postoperative mortality (odds ratio [OR], 2.41; 95\% confidence interval [CI], 1.54$3.71 ; P<.0001$ ), major adverse cardiac events (OR, 2.07; 95\% CI, 1.55-2.78; $P \leq .0001$ ), and prolonged mechanical ventilation (OR, 2.08; 95\% CI, 1.04-4.16; $P=.04)$ compared with patients without diastolic dysfunction among patients who underwent cardiovascular surgery. The odds of postoperative myocardial infarction (OR, 1.29; 95\% CI, 0.82-2.05; $P=.28$ ) and atrial fibrillation (OR, $2.67 ; 95 \%$ CI, $0.49-14.43 ; P=.25$ ) did not significantly differ between the 2 groups. Severity of preoperative diastolic dysfunction was associated with increased postoperative mortality (OR, 21.22; 95\% CI, 3.74-120.33; $P=.0006)$ for Grade 3 diastolic dysfunction compared with patients with normal diastolic function. Inclusion of left ventricular ejection fraction (LVEF) $<40 \%$ accompanying diastolic dysfunction did not further impact postoperative mortality $\left(P=.27 ; \mathrm{I}^{2}=18 \%\right)$ compared with patients with normal LVEF and diastolic dysfunction.

Conclusions: Presence of preoperative diastolic dysfunction was associated with greater postoperative mortality and major adverse cardiac events, regardless of LVEF. Mortality was significantly greater in grade III diastolic dysfunction. (J Thorac Cardiovasc Surg 2016;152:1142-53)

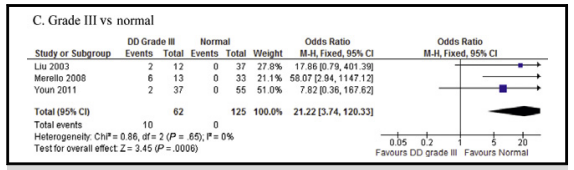

Postoperative mortality after cardiovascular surgery and grade III of diastolic dysfunction.

\section{Central Message}

Grade III diastolic dysfunction is associated with greater postoperative death after cardiovascular surgery, regardless of left ventricular ejection fraction.

\section{Perspective}

Underpowered studies and heterogeneity in measurement underestimate diastolic dysfunction (DD) in the perioperative period. We show increased perioperative mortality with greater grade of DD. This is not correctly predicted by current risk scoring systems. Limitations in understanding of DD and lack of specific therapy should not discourage its preoperative staging or preventive risk stratification.

See Editorial Commentary page 1154.
One in seven patients suffer a major complication or death after coronary artery bypass surgery (CABG), and the currently available cardiac surgery risk scores do not always

\footnotetext{
From the ${ }^{\mathrm{a}}$ Department of Hospital Medicine, Medicine Institute, ${ }^{\mathrm{b}}$ Department of Outcomes Research, Anesthesiology Institute, ${ }^{\mathrm{c}}$ Health Outcomes and Clinical Epidemiology Section, Department of Quantitative Health Sciences, Lerner Research Institute, ${ }^{\mathrm{f}}$ Medicine Institute Center for Value Based Care Research, and ${ }^{\mathrm{n}}$ Department of Thoracic and Cardiovascular Surgery, Cleveland Clinic; ${ }^{\mathrm{d}}$ School of Medicine, Universidad Peruana de Ciencias Aplicadas (UPC), Lima, Peru; ${ }^{\mathrm{e} D e p a r t m e n t}$ of Medicine, Case Western Reserve University, Cleveland, Ohio; ${ }^{\mathrm{g}}$ Department of Cardiovascular Medicine, University of Virginia, Charlottesville, Va; ${ }^{\mathrm{h}}$ Centro Nacional de Investigaciones Cardiovasculares (CNIC); ${ }^{\mathrm{i}}$ Department of Cardiology, Hospital Universitario 12 de Octubre; ${ }^{\mathrm{j}}$ Universidad Complutense de Madrid, Madrid, Spain; ${ }^{k}$ University of Connecticut School of Pharmacy, Storrs, Conn; ${ }^{1}$ Department of Medicine, Stanford Prevention Research
}

correctly predict these risks. $^{1-5}$ Recent literature emphasizes the adverse prognostic impact of diastolic dysfunction on postoperative outcomes, and many recent

Center, Stanford University School of Medicine, Stanford, Calif; and ${ }^{\mathrm{m}}$ Brigham and Women's Hospital Heart \& Vascular Center and Harvard Medical School, Boston, Mass.

Received for publication Jan 13, 2016; revisions received April 29, 2016; accepted for publication May 30, 2016; available ahead of print June 28, 2016.

Address for reprints: Adrian V. Hernandez, MD, PhD, School of Medicine, Universidad Peruana de Ciencias Aplicadas (UPC), Building F, Campus Villa, Av. Alameda de San Marcos Cuadra 2 s/n, Chorrillos, Lima 9, Peru (E-mail: adrianhernandezdiaz@gmail.com). $0022-5223 / \$ 36.00$

Copyright $(\subset 2016$ by The American Association for Thoracic Surgery http://dx.doi.org/10.1016/j.jtcvs.2016.05.057 


\section{Abbreviations and Acronyms \\ $\mathrm{AF} \quad=$ atrial fibrillation \\ $\mathrm{CABG}=$ coronary artery bypass surgery \\ $\mathrm{CAD}=$ coronary artery disease \\ EuroSCORE $=$ European System for Cardiac \\ Operative Risk Evaluation \\ LVEF = left ventricular ejection fraction \\ MACE $=$ major adverse cardiac events \\ MI = myocardial infarction \\ NOS $=$ Newcastle Ottawa Scale}

\section{Scanning this QR code will take} you to supplemental figures and tables for this article.

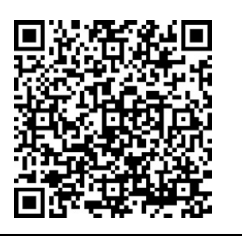

studies consistently rate severe diastolic dysfunction as the single most important determinant of postoperative outcome after cardiac surgery. Despite continuing controversies in the evaluation of diastolic dysfunction, recent advances in echocardiography have resulted in better assessment and reporting of diastolic dysfunction. The American College of Cardiology/American Heart Association/American Society of Echocardiography Appropriateness Criteria provide recommendations for echocardiography before noncardiac surgery but do not address the need for routine echocardiography before CABG. Among the echocardiographic criteria for risk scoring, the Society of Thoracic Surgeons uses left ventricular ejection fraction (LVEF) and significant valvular regurgitation/stenosis, whereas the European System for Cardiac Operative Risk Evaluation (EuroSCORE) includes LVEF and pulmonary arterial hypertension.

The current literature reporting postoperative outcomes after cardiac surgery in patients with varying degrees of diastolic dysfunction does not report postoperative mortality consistently as an independent outcome. Some of the reasons surrounding this observation could be mainly observational studies with low sample size or rare mortality outcomes that therefore had to be reported as part of a composite outcome of major adverse cardiac events. In addition, despite the increasing prevalence of diastolic heart failure, the majority of these patients do not happen to have severe or stage III diastolic dysfunction, thereby making it harder to assess its impact on postoperative outcomes.

We propose that diastolic dysfunction-especially if severe-can be an important predictor of postoperative outcomes and should be considered preoperatively for better risk stratification. To better evaluate postoperative outcomes after cardiovascular surgery, we conducted a systematic review and meta-analysis to investigate the effect of preoperative diagnosis of diastolic dysfunction on postoperative mortality and morbidity.

\section{METHODS}

We conducted a systematic review following the PRISMA (ie, Preferred Reporting Items for Systematic reviews and Meta-Analysis) guidelines. $^{6}$

\section{Search Strategy, Eligibility Criteria, and Study Selection}

Two authors independently identified the relevant articles by searching the following databases: PubMed, Cochrane Library, Web of Science, EMBASE, and Scopus until February 2016. The following search terminologies were used: (coronary artery bypass graft or CABG or cardiopulmonary bypass) and (diastolic function or diastolic dysfunction or impaired relaxation or pseudo normal filling or restrictive filling or diastolic filling or LV filling pressure or E/e or tissue Doppler) and (mortality or outcome). The search strategy for PubMed is available as an Online Data Supplement. The inclusion criteria were diastolic dysfunction on postoperative outcomes, including mortality and complications after cardiac surgery. Disagreements between these 2 authors were reviewed together and the decision was made on agreement. The 2 authors independently reviewed full text articles to determine whether the study can be included for the meta-analysis. We also reviewed the references of included articles.

\section{Data Extraction and Quality Assessment}

Two authors independently extracted relevant data from the studies on the standard data extraction sheet. Extracted data included definition of diastolic dysfunction, degree of diastolic dysfunction, type of surgery, exclusion criteria, primary and secondary outcomes, mode of echocardiogram, and follow-up period.

\section{Study Outcomes}

The primary study outcome was mortality. Secondary study outcomes were postoperative complications, which include major adverse cardiac events (MACE), prolonged mechanical ventilation, atrial fibrillation (AF), and myocardial infarction (MI).

\section{Statistical Analysis}

We performed meta-analysis with RevMan 5.3 (Cochrane Collaboration, Copenhagen, Denmark) and R 3.0.1 (www.r-project.org) using the Mantel-Haenszel fixed-effects model, given the scarcity of events. ${ }^{7}$ Random effects meta-analyses via the inverse variance method were performed otherwise. As sensitivity analyses for main analyses of mortality, 3 other methods were used: (1) natural weighting in random effects meta-analysis ${ }^{8}$; (2) Hartung-Knapp-Sidik-Jonkman method in random effects meta-analysis ${ }^{9}$; and (3) arcsine transformation of risk difference. ${ }^{10}$ For mortality, funnel plots were used to evaluate small study effects, and the Egger test was used to evaluate asymmetry of funnel plots. The Cochran $\chi^{2}$ test and the $\mathrm{I}^{2}$ test were used to assess between-study heterogeneity. Heterogeneity was considered statistically significant at $P<.1$, and substantial heterogeneity was defined as $\mathrm{I}^{2}>$ $50 \%$. Pooled odds ratios (ORs) were reported with $95 \%$ confidence intervals (CIs). 


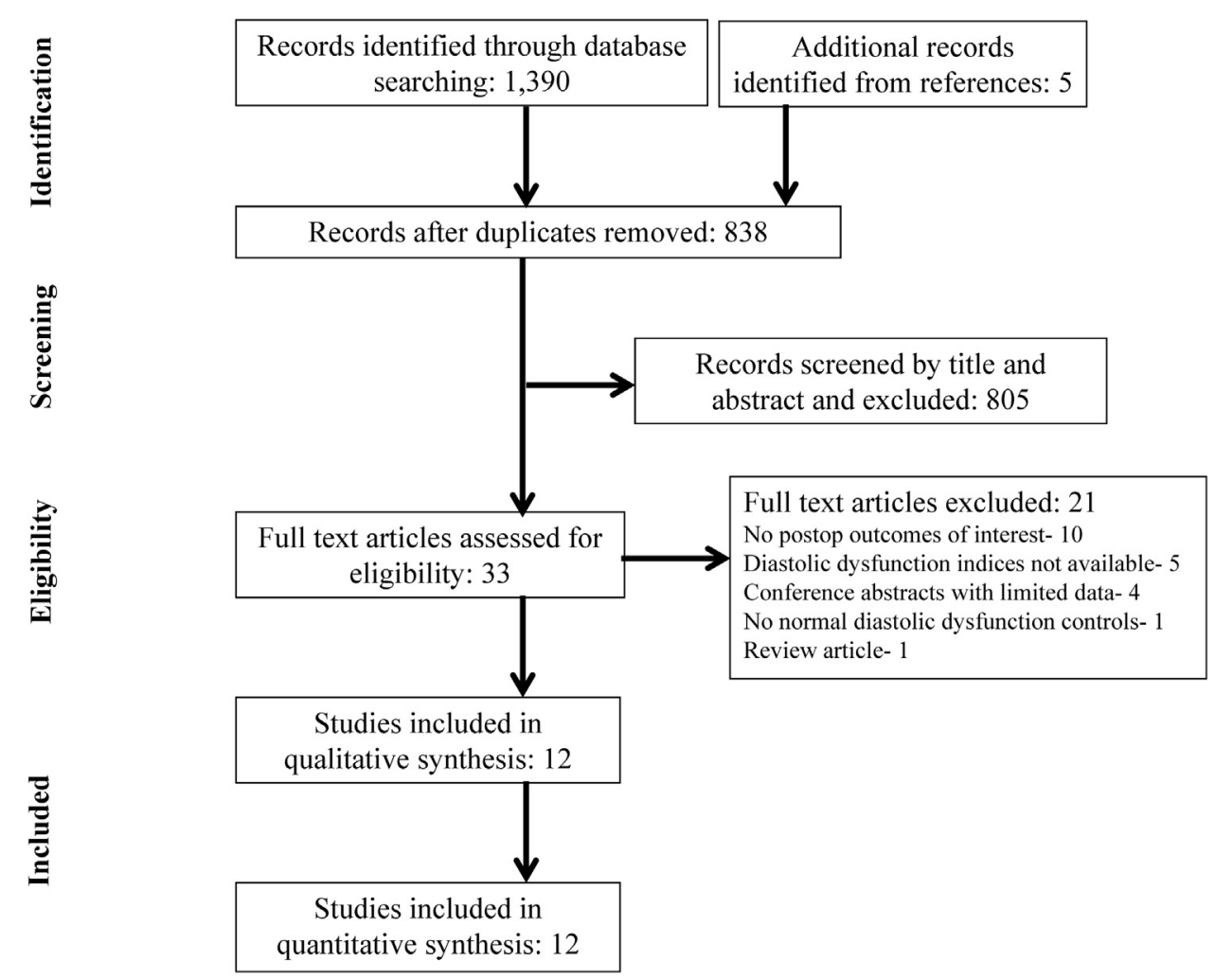

FIGURE 1. Study selection flow chart.

\section{Risk of Bias Evaluation}

Risk of bias of cohort studies was evaluated with the Newcastle Ottawa Scale (NOS). ${ }^{11}$ NOS scale evaluates the following criteria: (1) Selection: representativeness of the exposed cohort, selection of the nonexposed cohort, ascertainment of exposure, demonstration that outcome of interest was not present at start of study; (2) Comparability: comparability of cohorts on the basis of the design or analysis; and (3) Outcome: assessment of outcome; if the follow-up was follow-up long enough for outcomes to occur; adequacy of follow-up of cohorts. The NOS assessment was done per each cohort study and awarded with stars (1-4) for each of the 3 criteria (selection, comparability, outcomes). NOS scores $>7$ were considered high-quality studies, and NOS scores of 5-7 were considered moderate-quality studies. We also described when available, matching variables and confounders adjusted for per study; finally, we compared, when available, unadjusted and adjusted risks per outcome and study.

\section{RESULTS}

\section{Search Results}

A total of 838 articles were retrieved after excluding mutual inclusions; 651 articles from PubMed, 92 articles from Cochrane Library, 335 articles from Web of Science, 247 articles from Embase, and 65 articles from Scopus. The algorithm for the search strategy is summarized in Figure 1. After a complete review of the articles, 8 studies were selected for the analysis and 21 studies were excluded for the following reasons: diastolic dysfunction indices not available for 5 studies, abstract presented at conferences for 4 studies, postoperative outcomes of interest not reported for 10 studies, normal diastolic function not used for comparison in 1 study, and 1 review article. Five more studies were found eligible after references of the selected studies were reviewed, ${ }^{12-16}$ of which 1 could not be used for lack of requisite data. ${ }^{12}$

\section{Description of Studies}

The majority of patients were male, their mean age was 64.5 years, and most of them underwent CABG. Three studies reported patients exclusively undergoing offpump CABG, ${ }^{14,17,18}$ and 1 study had patients undergoing exclusive aortic or peripheral vascular surgery. ${ }^{15}$ Preoperative diastolic dysfunction was assessed by transthoracic echocardiography, ${ }^{13,17-23}$ transesophageal echocardiography, ${ }^{15,24}$ and left heart catheterization. ${ }^{14,16}$ Mean follow-up ranged between inpatient postoperative period to 3.2 years (Table 1). Postoperative outcomes and confounding variables per study are described in Table 2.

\section{Risk of Bias of Included Studies}

Key points of risk of bias were assessed with the NOS (Table 3). All studies clearly identified and excluded the conditions that would be considered as important 
confounders in measurement of diastolic dysfunction (Table 2). Overall, all studies clearly identified the study population, reported the main outcome, and scored well on other quality items. No selective loss of follow-up was reported. A summary of unadjusted and adjusted outcome risk estimates by presence or grade of diastolic dysfunction and per study are reported in Table E1. All studies were observational; we did not identify any trials. Our analyses provided no indication of publication bias. No asymmetry of funnel plot was noted (Figure E1), and the formal test of asymmetry was not significant (Egger test, $P=.3$ ).

\section{Effect of Diastolic Dysfunction on Postoperative Mortality and MACE}

A total of 10 studies $(n=6911)^{13-20,23,24}$ investigated the impact of preoperative diastolic dysfunction on postoperative mortality independent of other complications. Six studies ${ }^{13-16,19,24}$ reported postoperative or in-hospital mortality, 2 studies ${ }^{17,20}$ reported mortality up to 30 days, 1 study reported mortality from 30 days to 1 year, ${ }^{18}$ and another 1 up to 23 months. ${ }^{23}$ To stay consistent that only postoperative outcomes were reported, we only used the 30-day data reported in the study by Lee and colleagues ${ }^{18}$ and also analyzed mortality by excluding the study by Liu and colleagues. ${ }^{23}$ A total of $51.3 \%$ (3546/6911) of patients had diastolic dysfunction. Eighty-seven $(2.45 \%)$ patients died among the 3546 patients with diastolic heart failure compared with $43(1.28 \%)$ deaths among 3365 patients with normal diastolic function (Table 2). Preoperative diagnosis of diastolic dysfunction was associated with increased postoperative mortality after cardiac surgery (OR, 2.41; 95\% CI, $1.57-3.71 ; P<.001 ; \mathrm{I}^{2}=0$ ) (Figure 2). Exclusion of the study by Liu and colleagues ${ }^{23}$ did not change the overall results $(P<.0001)$. The results of sensitivity analysis with the arcsine transformed risk difference (at risk difference $0.0625 ; 95 \% \mathrm{CI}$, $0.0326-0.0915 ; P=.001$ ) were concordant with the results from the main analysis; risk difference $0.0647 ; 95 \% \mathrm{CI}$, $0.0324-0.0971 ; P<.001$ when the paper by Liu and colleagues was excluded.

We also investigated the effect of severity of diastolic dysfunction on postoperative mortality. Of the studies reporting postoperative mortality, only 3 studies $^{17,20,23}$ provided complete gradation of diastolic dysfunction and comparisons with normal diastolic function. Two studies $^{13,19}$ used stage I diastolic dysfunction patients only for comparison with those with normal diastolic function. Of a total of 1424 patients with any degree of diastolic dysfunction, $62(4.35 \%)$ had severe diastolic dysfunction or restrictive filling. Greater postoperative mortality was noted with increasing severity of diastolic dysfunction, but it was statistically significant only in the group with severe diastolic dysfunction or restrictive filling (OR, 21.22; 95\% CI, 3.74-120.33; $P=.006$;
$\mathrm{I}^{2}=0$ ) (Figure 3). Percentage mortality was $1.12 \%$ in delayed relaxation phase; $2.39 \%$ in pseudo-normal filling, and $16 \%$ in restrictive filling group. Exclusion of the paper by Liu and colleagues ${ }^{23}$ did not change the overall results, $\mathrm{Z}=2.93(P=.003)$.

Diastolic dysfunction on preoperative echocardiogram was associated with a greater incidence of MACE (OR, $2.07 ; 95 \% \mathrm{CI}, 1.55-2.78 ; P<.0001)$ and prolonged mechanical ventilation $(\mathrm{OR}, 2.08 ; 95 \% \mathrm{CI}, 1.04-4.16$; $P=.04$ ) (Figure 4) among patients who underwent cardiac surgery. Youn and colleagues ${ }^{17}$ and Lee and colleagues ${ }^{18}$ reported both postoperative mortality and MACE and death was included in MACE; however, diastolic dysfunction continued to be associated with MACE after these studies were excluded in a sensitivity analysis (OR, 1.94; $95 \%$ CI, 1.32-2.86; $P=.0007 ; \mathrm{I}^{2}=0 \%$ ) (Figure E2). The severity of diastolic dysfunction also was associated with a greater incidence of MACE but was statistically significant only in grade II (OR, 1.84; $95 \%$ CI, 0.99-3.43; $\left.P=.05 ; \mathrm{I}^{2}=0 \%\right)$ and grade III severity classes (OR, 4.69; 95\% CI, 2.52-8.72; $P<.0001$ ) (Figure E3). Postoperative MI and AF did not differ between the 2 groups (Figure 4).

\section{Subgroup Analyses}

The following subgroups were analyzed: patients with diastolic dysfunction with normal LVEF or mean LVEF $>40$ versus patients with diastolic dysfunction with LVEF <40; types of cardiac surgery (CABG + valve vs CABG only), and diastolic dysfunction as reported by transthoracic echocardiography or transesophageal echocardiography. The excess in postoperative mortality associated with diastolic function was noted in both the groups that included patients with normal LVEF as well as with reduced LVEF (OR, 2.16; 95\% CI, 1.35-3.45 vs OR, $4.31 ; 95 \%$ CI, 1.39-13.38; $P$ for interaction $=.27$; $\mathrm{I}^{2}=17.8 \%$; Figure E4). Postoperative outcomes were similar regardless of whether diastolic dysfunction was identified and quantified by transthoracic (OR, 2.42; 95\% CI, 1.54-3.80) versus transesophageal echocardiogram (OR, 2.34; 95\% CI, 0.56-9.79); $P$ for interaction $=.97$; $\mathrm{I}^{2}=0 \%$ (Figure E5) or by the type of surgery: CABG, OR $2.42,95 \%$ CI $1.54-3.80$ versus CABG + valve surgery, OR $12.57,95 \%$ CI $0.58-274.49$ versus vascular surgery, OR $1.20,95 \%$ CI $0.21-6.70 ; P$ for interaction $=.42 ; \mathrm{I}^{2}=0 \%$ (Figure E6). Subgroup analysis for outcomes by age was not possible.

\section{DISCUSSION}

Within the limitations associated with assessing the true impact of diastolic dysfunction on perioperative outcomes after cardiovascular surgery, our analysis reveals greater postoperative mortality in patients with diastolic dysfunction. In a subgroup analysis, this effect was seen 
TABLE 1. Characteristics of included studies

\begin{tabular}{|c|c|c|c|c|c|c|c|c|}
\hline $\begin{array}{c}\text { First author, } \\
\text { year of } \\
\text { publication }\end{array}$ & $\begin{array}{l}\text { Type of } \\
\text { surgery }\end{array}$ & Sample size & $\begin{array}{c}\text { Age, } y \\
\text { mean (SD) }\end{array}$ & Males (\%) & $\begin{array}{c}\text { BMI, } \\
\text { mean (SD) }\end{array}$ & Obesity, \% & HTN, \% & Diabetes, $\%$ \\
\hline Afilalo, 2013 & CABG & $\begin{array}{l}\text { No DD: } 237 \\
\text { DD: } 395\end{array}$ & $67.2(11.1)$ & 77.2 & $28.8(5.2)$ & NS & NS & 37.5 \\
\hline Bagheri, 2010 & CABG & $\begin{array}{l}\text { No DD: } 361 \\
\text { DD: } 27\end{array}$ & $\begin{array}{l}\text { No DD: } 57.7(9.5) \\
\text { DD: } 61.2(9.5)\end{array}$ & 68.0 & NS & NS & $\begin{array}{l}\text { No DD: } 45.2 \\
\text { DD: } 40.7\end{array}$ & $\begin{array}{l}\text { No DD: } 23.1 \\
\text { DD: } 29.6\end{array}$ \\
\hline Bernard, 2001 & $\begin{array}{l}\text { On-pump CABG } \\
(78.8 \%) ; \\
\text { combined } \\
\text { procedures, } \\
\text { valvular surgery } \\
\text { and reoperations } \\
(21.2 \%)\end{array}$ & $\begin{array}{l}\text { No DD: } 46 \\
\text { DD: } 20\end{array}$ & $\begin{array}{l}\text { No DD: } 63(8) \\
\text { DD: } 67(8)\end{array}$ & 66.7 & $\begin{array}{c}\text { No DD: } \\
27.3 \\
\text { DD: } 25.3\end{array}$ & NS & $\begin{array}{l}\text { No DD: } 47.8 \\
\text { DD: } 55.0\end{array}$ & $\begin{array}{l}\text { No DD: } 19.6 \\
\text { DD: } 40.0\end{array}$ \\
\hline $\begin{array}{l}\text { Değirmencioğlu, } \\
2013\end{array}$ & CABG & $\begin{array}{l}\text { No DD: } 168 \\
\text { DD: } 304\end{array}$ & $\begin{array}{l}\text { No DD: } 58.1(0.8) \\
\text { DD: } 62.4(0.5)\end{array}$ & 75.6 & $\begin{array}{l}\text { No DD: } \\
29.1(4.3) \\
\text { DD: } \\
29.1(4.4)\end{array}$ & NS & $\begin{array}{c}\text { No DD: } 71.4 \\
\text { DD: } 80.6\end{array}$ & $\begin{array}{l}\text { No DD: } 35.1 \\
\text { DD: } 42.9\end{array}$ \\
\hline Lee, 2012 & Off-pump CABG & $\begin{array}{l}\text { No DD: } 744 \\
\text { DD: } 304\end{array}$ & $\begin{array}{l}\text { No DD: } 63 \\
\text { DD: } 67\end{array}$ & 75.4 & $\begin{array}{l}\text { No DD: } \\
24.6(2.7) \\
\text { DD: } \\
24.9(3.0)\end{array}$ & NS & $\begin{array}{c}\text { No DD: } 58.9 \\
\text { DD: } 74.3\end{array}$ & $\begin{array}{l}\text { No DD: } 40.2 \\
\text { DD: } 62.2\end{array}$ \\
\hline Liu, 2003 & CABG & $\begin{array}{l}\text { No DD: } 37 \\
\text { DD: } 65\end{array}$ & $\begin{array}{l}\text { No DD: } 66(9) \\
\text { DD: } 66.1(8.7)\end{array}$ & 74.5 & NS & NS & $\begin{array}{l}\text { No DD: } 35.0 \\
\text { DD: } 36.9\end{array}$ & $\begin{array}{l}\text { No DD: } 38.0 \\
\text { DD: } 41.5\end{array}$ \\
\hline Matyal, 2009 & $\begin{array}{l}\text { Elective } \\
\text { vascular } \\
\text { surgery } \\
\text { (aortic or } \\
\text { peripheral) }\end{array}$ & $\begin{array}{l}\text { No DD: } 80 \\
\text { DD: } 134\end{array}$ & 70.8 & 63.0 & NS & NS & 85.0 & 76.2 \\
\hline $\begin{array}{l}\text { Melduni, } \\
2011\end{array}$ & $\begin{array}{l}\text { CABG }(45.6 \%) \\
\text { valvular surgery, } \\
\text { and CABG }+ \\
\text { valvular surgery } \\
(54.4 \%)\end{array}$ & $\begin{array}{l}\text { No DD: } 79 \\
\text { DD: } 272\end{array}$ & 66.7 (13.6) & 67.2 & $28.2(5.2)$ & NS & 74.1 & 25.9 \\
\hline Merello, 2008 & On-pump CABG & $\begin{array}{l}\text { No DD: } 33 \\
\text { DD: } 158\end{array}$ & $\begin{array}{c}\text { No DD: } 59.1(9.4) \\
\text { DD: } 63.9(8.6)\end{array}$ & 72.3 & $\begin{array}{c}\text { No DD: } \\
27.4 \\
\text { DD: } 26.7\end{array}$ & $\begin{array}{l}\text { No DD: } 12.1 \\
\text { DD: } 13.9\end{array}$ & $\begin{array}{c}\text { No DD: } 69.7 \\
\text { DD: } 81.0\end{array}$ & $\begin{array}{l}\text { No DD: } 33.3 \\
\text { DD: } 32.9\end{array}$ \\
\hline Salem, 2006 & $\begin{array}{l}\text { CABG }(87.1 \%) ; \\
\text { valvular surgery, } \\
\text { and CABG }+ \\
\text { valvular surgery } \\
(12.9 \%)\end{array}$ & $\begin{array}{l}\text { No DD: } 1051 \\
\text { DD: } 1279\end{array}$ & 63.2 & 63.3 & $27(5)$ & NS & 45.3 & 18.3 \\
\hline Sastry, 2010 & Off-pump CABG & $\begin{array}{l}\text { No DD: } 790 \\
\text { DD: } 54\end{array}$ & $\begin{array}{l}\text { No DD: } 64.7 \\
\text { DD: } 66.2\end{array}$ & 80.0 & $\begin{array}{c}\text { No DD: } \\
28.2 \\
\text { DD: } 28.2\end{array}$ & NS & $\begin{array}{l}\text { No DD: } 57.6 \\
\text { DD: } 57.4\end{array}$ & $\begin{array}{l}\text { No DD: } 21.3 \\
\text { DD: } 22.2\end{array}$ \\
\hline Youn, 2011 & Off-pump CABG & $\begin{array}{l}\text { No DD: } 55 \\
\text { DD: } 1201\end{array}$ & $\begin{array}{c}\text { No DD: } 54.9(9.9) \\
\text { DD: } 63.5(8.4)\end{array}$ & 71.8 & NS & $\begin{array}{l}\text { No DD: } 5.5 \\
\text { DD: } 2.8\end{array}$ & $\begin{array}{l}\text { No DD: } 43.6 \\
\text { DD: } 66.5\end{array}$ & $\begin{array}{l}\text { No DD: } 20.0 \\
\text { DD: } 42.5\end{array}$ \\
\hline
\end{tabular}

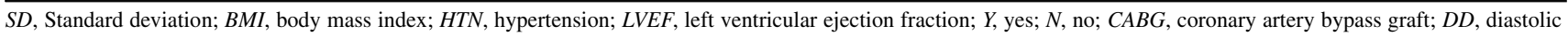
dysfunction; $N S$, not stated; $T T E$, transthoracic echocardiogram; $T E E$, transesophageal echocardiogram; $C P B$, cardiopulmonary bypass; $A F$, atrial fibrillation; $b p m$, beats per minute; $I C U$, intensive care unit; $M R$, mitral regurgitation; $M I$, myocardial infarction; $C H F$, congestive heart failure; $C H D$, congenital heart disease; $L V E D P$, left ventricular enddiastolic pressure; $P D A$, patent ductus arteriosus; $C B P$, cardiopulmonary bypass; $T D I$, tissue Doppler imaging. *Mean (standard deviation). 
TABLE 1. Continued

\begin{tabular}{|c|c|c|c|c|}
\hline $\begin{array}{c}\text { Preoperative diastolic } \\
\text { dysfunction indices } \\
\text { measured by }\end{array}$ & $\begin{array}{l}\text { Follow-up } \\
\text { period }\end{array}$ & $\begin{array}{c}\text { Study enrolled } \\
\text { normal LVEF } \\
\text { patients } \\
\text { only }(\mathrm{Y} / \mathrm{N})\end{array}$ & Study exclusion criteria & Primary outcome(s) \\
\hline TTE & $3.2 \mathrm{y}$ & $\mathrm{N}$ & $\begin{array}{l}\text { Echocardiographic reports from an outside center; } \\
\text { digital echocardiographic images not retrievable } \\
\text { because of technical reasons }\end{array}$ & $\begin{array}{l}\text { In-hospital mortality or } \\
\text { major morbidity }\end{array}$ \\
\hline Echocardiography & Postoperative & $\mathrm{Y}$ & $\begin{array}{l}\mathrm{LVEF}<50 \% \text {; CABG with valve surgery or } \mathrm{AF} \\
\text { ablation therapy }\end{array}$ & NS \\
\hline TEE & Postoperative & $\mathrm{N}$ & $\begin{array}{l}\text { Cardiac surgery without CPB; heart rate more than } \\
100 \text { bpm; persistent arrhythmia; if they required } \\
\text { a pacemaker }\end{array}$ & $\begin{array}{l}\text { Mortality and surgical ICU } \\
\text { length of stay }\end{array}$ \\
\hline $\begin{array}{l}\text { Mitral inflow waves, } \\
\text { tissue Doppler imaging } \\
\text { and pulmonary vein flow } \\
\text { analysis by echocardiography }\end{array}$ & $30 \mathrm{~d}$ & $\mathrm{Y}$ & $\begin{array}{l}\text { LVEF }<50 \% \text {; baseline rhythm other than sinus; } \\
\text { moderate or severe valvular dysfunction; moderate } \\
\text { or severe diastolic dysfunction }\end{array}$ & $\begin{array}{l}\text { Perioperative morbidity } \\
\text { and mortality }\end{array}$ \\
\hline TTE & $1 \mathrm{y}$ & $\mathrm{N}$ & $\begin{array}{l}\text { AF; emergency surgery; concomitant valvular or aortic } \\
\text { surgery; participation in another trial during that } \\
\text { period; grade } \geq 2 / 4 \text { MR on echocardiography; those } \\
\text { with a preoperative inotrope, intra-aortic balloon } \\
\text { pump or ventricular assist device support }\end{array}$ & Major adverse cardiac events \\
\hline $\begin{array}{l}\text { Trans-mitral flow, pulmonary } \\
\text { venous flow by Doppler } \\
\text { echocardiography }\end{array}$ & $29(20) \mathrm{mo}^{*}$ & $\mathrm{~N}$ & $\begin{array}{l}\text { AF; sinus tachycardia; valvular aortic stenosis; } \\
\text { emergency operation for acute MI; grade }>2 / 4 \\
\text { MR; chronic renal failure }\end{array}$ & Cardiac events \\
\hline TEE & Postoperative & $\mathrm{N}$ & $\begin{array}{l}\text { Emergency surgery, surgery under regional anesthesia; } \\
\text { severe vascular disorders; contraindications to TEE }\end{array}$ & $\begin{array}{l}\text { MI, CHF, significant } \\
\text { arrhythmia, prolonged } \\
\text { intubation, renal failure, } \\
\text { and death }\end{array}$ \\
\hline TTE & $30 \mathrm{~d}$ & $\mathrm{~N}$ & $\begin{array}{l}\text { History of AF, Cox maze, or radiofrequency ablation } \\
\text { procedure for atrial arrhythmias; CHD; moderate } \\
\text { or severe mitral stenosis; incomplete diastolic } \\
\text { function assessment }\end{array}$ & Postoperative AF \\
\hline $\begin{array}{l}\text { Pulsed Doppler mitral filling } \\
\text { flow, left ventricular diastolic } \\
\text { filling patterns measured } \\
\text { by echocardiography }\end{array}$ & $30 \mathrm{~d}$ & $\mathrm{~N}$ & $\begin{array}{l}\text { AF; off-pump CABG; CABG associated with valve } \\
\text { surgery; severe mitral or aortic valvulopathy; } \\
\text { implanted pacemaker }\end{array}$ & $\begin{array}{l}\text { Postoperative morbidity } \\
\text { and mortality }\end{array}$ \\
\hline LVEDP & Postoperative & $\mathrm{N}$ & $\begin{array}{l}\text { Off-pump CABG; surgery of the descending aorta } \\
\text { or PDA }\end{array}$ & In-hospital mortality \\
\hline LVEDP & Postoperative & $\mathrm{N}$ & Revascularization on CBP & $\begin{array}{l}\text { In-hospital mortality and ICU } \\
\text { length of stay }\end{array}$ \\
\hline TTE & $30 \mathrm{~d}$ & $\mathrm{~N}$ & $\begin{array}{l}\text { AF; grade more than } 2 / 4 \mathrm{MR} \text {; need for dialysis; urgent/ } \\
\text { emergency operation; undetermined diastolic function } \\
\text { including sinus tachycardia; aortic stenosis; on-pump } \\
\text { conversion; diastolic function not assessed by TDI }\end{array}$ & Major adverse cardiac events \\
\hline
\end{tabular}


TABLE 2. Confounding variables identified and postoperative outcomes

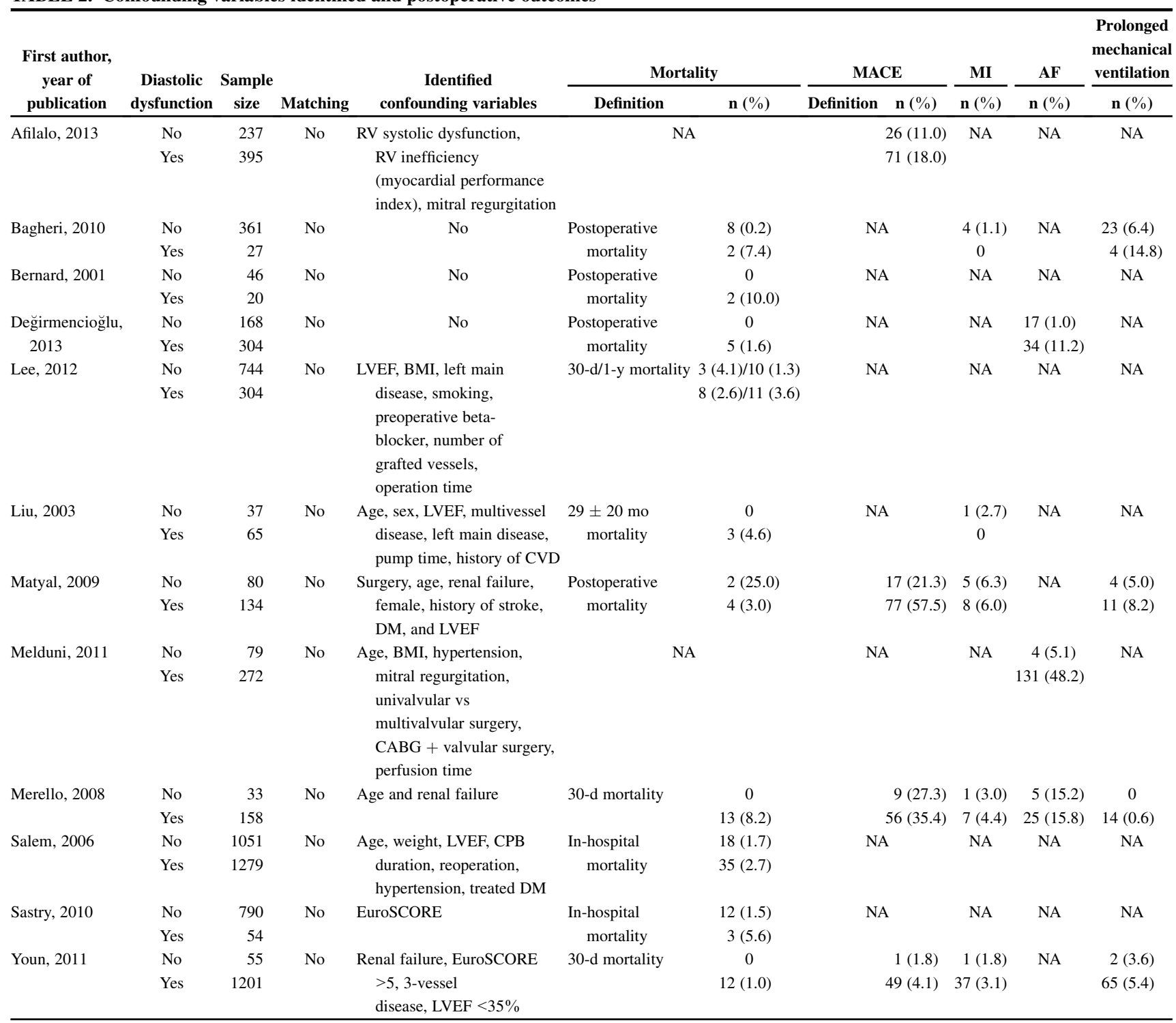

$M A C E$, Major adverse cardiovascular events; $M I$, myocardial infarction; $A F$, atrial fibrillation; $R V$, right ventricle; $N A$, not available; $L V E F$, left ventricular ejection fraction; $B M I$, body mass index; $C V D$, cerebrovascular disease; $D M$, diabetes mellitus; $C A B G$, coronary artery bypass graft; $C P B$, cardiopulmonary bypass; EuroSCORE, European system for cardiac operative risk evaluation.

regardless of associated left ventricular systolic dysfunction and appeared to vary with the severity of diastolic dysfunction, reaching statistical significance in grade III diastolic dysfunction. Patients with diastolic dysfunction also were more likely to experience major adverse cardiac events and prolonged mechanical ventilation. No difference was noted in the incidence of postoperative MI and AF between the 2 groups.

It has been known from very early studies that greater end-diastolic pressures among patients undergoing CABG were associated with greater preoperative mortality. ${ }^{25}$ Among patients receiving surgery for coronary artery disease (CAD) or aortic stenosis, the incidence of left ventricular diastolic dysfunction ranges between $44 \%$ and $75 \% .{ }^{26-28}$ In our study, the difference between mortality in groups with delayed relaxation (stage I diastolic dysfunction) and pseudonormal pattern (stage II diastolic dysfunction) was not statistically significant compared with patients with normal diastolic function, despite relatively greater prevalence. ${ }^{17,20,23}$

One possible explanation could be that with revascularization the milder forms of diastolic dysfunction improve, resulting in no difference in outcomes compared with those with normal diastolic function. Vaskelyte and colleagues ${ }^{29}$ showed that LV wall motion score did not improve in $67 \%$ of patients with restrictive filling compared with 
TABLE 3. NOS risk of bias scale for included cohort studies

\begin{tabular}{|c|c|c|c|c|c|c|c|c|c|}
\hline \multirow[b]{2}{*}{ Studies } & \multicolumn{4}{|c|}{ Selection } & \multirow[b]{2}{*}{ Comparability } & \multicolumn{3}{|c|}{ Outcome } & \multirow[b]{2}{*}{$\begin{array}{l}\text { Total } \\
\text { score }\end{array}$} \\
\hline & $\begin{array}{c}\text { Representativeness } \\
\text { of the exposed } \\
\text { cohort }\end{array}$ & $\begin{array}{c}\text { Selection of the } \\
\text { nonexposed } \\
\text { cohort }\end{array}$ & $\begin{array}{c}\text { Ascertainment } \\
\text { of exposure }\end{array}$ & $\begin{array}{c}\text { Outcome of } \\
\text { interest not } \\
\text { present at start } \\
\text { of study }\end{array}$ & & $\begin{array}{l}\text { Assessment } \\
\text { of outcome }\end{array}$ & $\begin{array}{c}\text { Adequacy } \\
\text { of duration } \\
\text { of follow-up }\end{array}$ & $\begin{array}{c}\text { Adequacy of } \\
\text { completeness } \\
\text { of follow-up }\end{array}$ & \\
\hline Afilalo, 2013 & $*$ & $*$ & $*$ & $*$ & $\begin{array}{c}*(\mathrm{RV} \text { systolic } \\
\text { dysfunction })\end{array}$ & $*$ & $*$ & $*$ & 8 \\
\hline Bagheri, 2010 & $*$ & $*$ & * & * & & * & $*$ & $*$ & 7 \\
\hline Bernard, 2001 & $*$ & * & $*$ & * & $* *($ age, $\mathrm{CPB})$ & * & * & * & 9 \\
\hline $\begin{array}{l}\text { Değirmencioğlu, } \\
2013\end{array}$ & * & * & * & * & & * & * & * & 7 \\
\hline Lee, 2012 & * & * & * & * & $*(\mathrm{LVEF})$ & * & * & * & 8 \\
\hline Liu, 2003 & * & * & $*$ & * & $* *$ (age, LVEF) & * & * & * & 9 \\
\hline Matyal, 2009 & $*$ & $*$ & * & * & $* *$ (age, LVEF) & * & * & $*$ & 9 \\
\hline Melduni, 2011 & * & * & * & * & $* *($ age, hypertension $)$ & * & * & * & 9 \\
\hline Merello, 2008 & * & * & * & * & $* *($ age, renal failure $)$ & * & * & * & 9 \\
\hline Salem, 2006 & $*$ & * & $*$ & * & $* *$ (age, LVEF) & $*$ & * & $*$ & 9 \\
\hline Sastry, 2010 & $*$ & $*$ & $*$ & * & $* *$ (EuroSCORE) & * & $*$ & * & 9 \\
\hline Youn, 2011 & * & * & * & * & $* *$ (EuroSCORE) & * & * & * & 9 \\
\hline
\end{tabular}

Each asterisk represents a score of 1 point. NOS scores $>7$ were considered high-quality studies, and NOS scores of 5-7 were considered moderate-quality studies. $N O S$, Newcastle Ottawa Scale; $R V$, right ventricle; $C P B$, cardiopulmonary bypass time; $L V E F$, left ventricular ejection fraction; EuroSCORE, European system for cardiac operative risk evaluation.

$42 \%$ or $43 \%$ of patients with stage I or II diastolic dysfunction respectively, in the early postoperative period. Despite similar degrees of systolic dysfunction (LVEF $<35 \%)$ and severity of CAD (93\% patients had previous MI and 3-vessel disease), patterns of LV diastolic filling varied, perhaps based on the duration of CAD, and for the most part determined postoperative mortality. Myocyte relaxation during diastole is an energy-dependent process and thus sensitive to impaired perfusion. ${ }^{30}$ Oppizzi and colleagues $^{31}$ reported that diastolic dysfunction persisted during stay in the intensive care unit, and 3 months after CABG it normalized in $85 \%$ of patients, and earlier studies have reported improvements beginning as early as 1 week. $^{32}$ These studies are limited by less-than-optimal measurement tools for diastolic dysfunction. For these reasons, some have questioned the utility of cardiac surgery in patients with severe diastolic dysfunction, therefore making preoperative evaluation imperative in these patients.

Mortality and other adverse outcomes associated with diastolic dysfunction are not predicted adequately and actually exceeded that predicted by traditional risk scoring

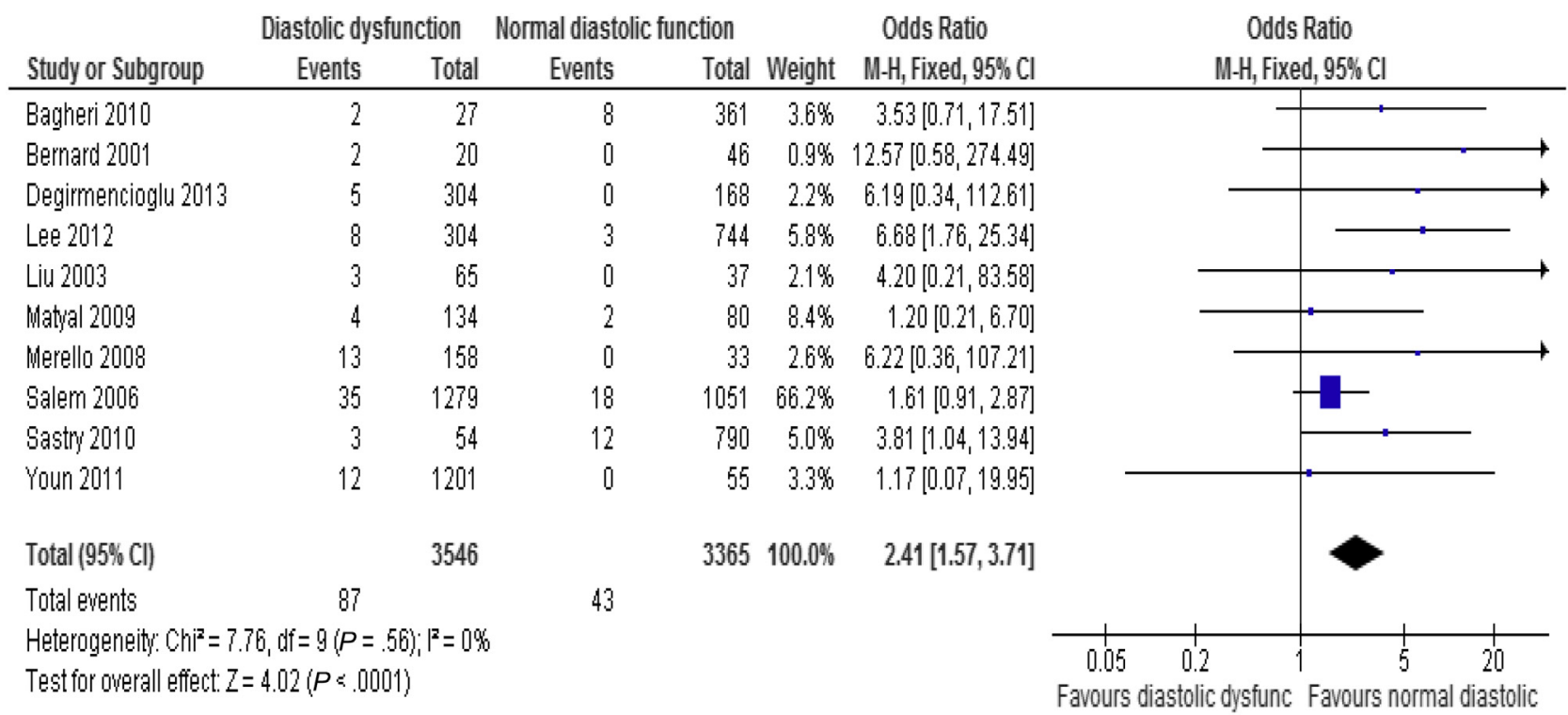

FIGURE 2. Mortality after cardiovascular surgery in patients with and without diastolic dysfunction. $C I$, Confidence interval; $M-H$, Mantel-Haenszel. 
Grade I vs normal

\begin{tabular}{|c|c|c|c|c|c|c|c|c|c|}
\hline Study or Subgroup & \multicolumn{2}{|c|}{ DD Grade I } & \multicolumn{2}{|c|}{ Normal } & \multicolumn{2}{|r|}{ Odds Ratio } & \multicolumn{2}{|c|}{$\begin{array}{c}\text { Odds Ratio } \\
\text { M-H, Fixed, } 95 \% \mathrm{Cl}\end{array}$} & \\
\hline Liu 2003 & 0 & 29 & 0 & 37 & & Not estimable & & & \\
\hline Merello 2008 & 5 & 129 & 0 & 33 & $44.7 \%$ & $2.96[0.16,54.88]$ & & 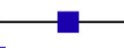 & \\
\hline Youn 2011 & 8 & 995 & 0 & 55 & $55.3 \%$ & $0.96[0.05,16.77]$ & & & \\
\hline Total $(95 \% \mathrm{Cl})$ & & 1153 & & 125 & $100.0 \%$ & $1.85[0.24,14.32]$ & & & \\
\hline Total events & 13 & & 0 & & & & & & \\
\hline $\begin{array}{l}\text { Heterogeneity: Chiz } \\
\text { Test for overall effec }\end{array}$ & $\begin{array}{l}0.30, \mathrm{df}= \\
Z=0.59\end{array}$ & $\begin{array}{l}1(P= \\
P=55\end{array}$ & $.58 ; ;\left.\right|^{2}=$ & & & & \begin{tabular}{cc|} 
& 1 \\
0.05 & 0.2 \\
Favours DD grade I
\end{tabular} & $\begin{array}{r}5 \\
\text { Favours }\end{array}$ & $\begin{array}{r}20 \\
\text { ormal }\end{array}$ \\
\hline
\end{tabular}

Grade II vs normal

\begin{tabular}{|c|c|c|c|c|c|c|c|c|}
\hline \multirow[b]{2}{*}{ Study or Subgroup } & \multicolumn{2}{|c|}{ DD Grade II } & \multicolumn{2}{|c|}{ Normal } & \multirow{3}{*}{$\frac{\text { Weight }}{26.7 \%}$} & \multirow{3}{*}{$\begin{array}{c}\text { Odds Ratio } \\
\text { M-H, Fixed, 95\% Cl } \\
4.79[0.19,122.47]\end{array}$} & \multirow{2}{*}{\multicolumn{2}{|c|}{$\begin{array}{c}\text { Odds Ratio } \\
\text { M-H, Fixed, } 95 \% \mathrm{Cl}\end{array}$}} \\
\hline & Events & Total & Events & Total & & & & \\
\hline Liu 2003 & 1 & 24 & 0 & 37 & & & & $=$ \\
\hline Merello 2008 & 2 & 16 & 0 & 33 & $20.3 \%$ & $11.55[0.52,255.97]$ & & \\
\hline Youn 2011 & 2 & 169 & 0 & 55 & $53.0 \%$ & $1.66[0.08,35.03]$ & & \\
\hline Total $(95 \% \mathrm{Cl})$ & & 209 & & 125 & $100.0 \%$ & $4.50[0.74,27.51]$ & & \\
\hline Total events & 5 & & 0 & & & & & \\
\hline $\begin{array}{l}\text { Heterogeneity: } \mathrm{Chi}^{2}= \\
\text { Test for overall effect }\end{array}$ & $\begin{array}{l}0.77, \mathrm{df}= \\
Z=1.63\end{array}$ & $\begin{array}{l}2(P= \\
P=.10\end{array}$ & 68 & & & & \begin{tabular}{cc|}
0.05 & 0.2 \\
Favours & DD grade II
\end{tabular} & $\begin{array}{cc}5 & 20 \\
\text { Favours Normal }\end{array}$ \\
\hline
\end{tabular}

Grade III vs normal DD Grade III Normal Odds Ratio

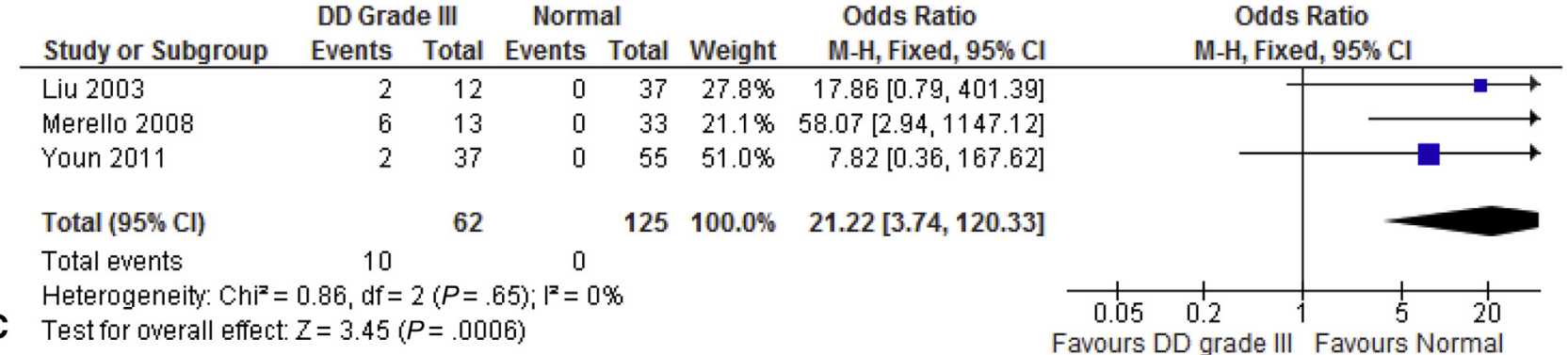

FIGURE 3. Postoperative mortality after cardiovascular surgery and grade of diastolic dysfunction: A, grade I versus normal; B, grade II versus normal; C, grade III versus normal. $D D$, Diastolic dysfunction; $M-H$, Mantel-Haenszel; $C I$, confidence interval.

systems such as EuroSCORE and Parsonnet in the pseudonormal relaxation and restrictive filling subgroups. ${ }^{23,33}$ These risk scores vastly underestimate the mortality associated with diastolic dysfunction but, as expected, are good at predicting mortality associated with other extracardiac conditions. One reason is that there is scarce evidence of diastolic dysfunction as an independent predictor of adverse outcomes, especially mortality. Sastry and colleagues ${ }^{14}$ showed that further risk stratification of mortality by EuroSCORE is augmented by additional use of left ventricular end-diastolic pressure, especially greater than $20 \mathrm{~mm} \mathrm{Hg}$ and the leftward shift of the curve approaches actual mortality (area under ROC increased from 0.70 to 0.78 ).

Our study has several limitations. The impact of diastolic dysfunction on mortality, MACE, and other outcomes is unadjusted for other factors that influence diastolic function, particularly in the perioperative period. Most studies, however, excluded patients with confounders for ventricular preload, namely atrial arrhythmias, moderate-to-severe valvular dysfunction, and chronic renal failure (Table 1) but this amounts up to at 


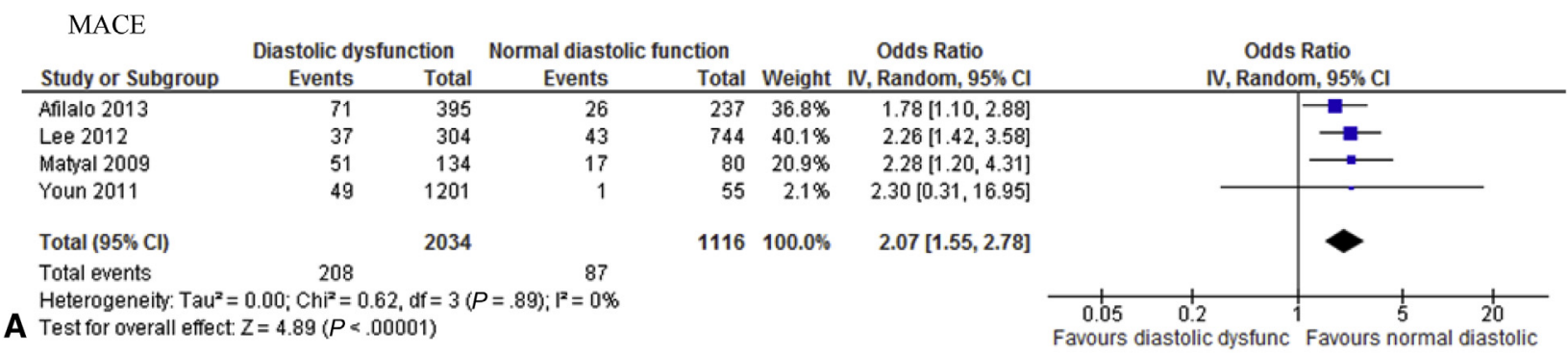

Myocardial infarction

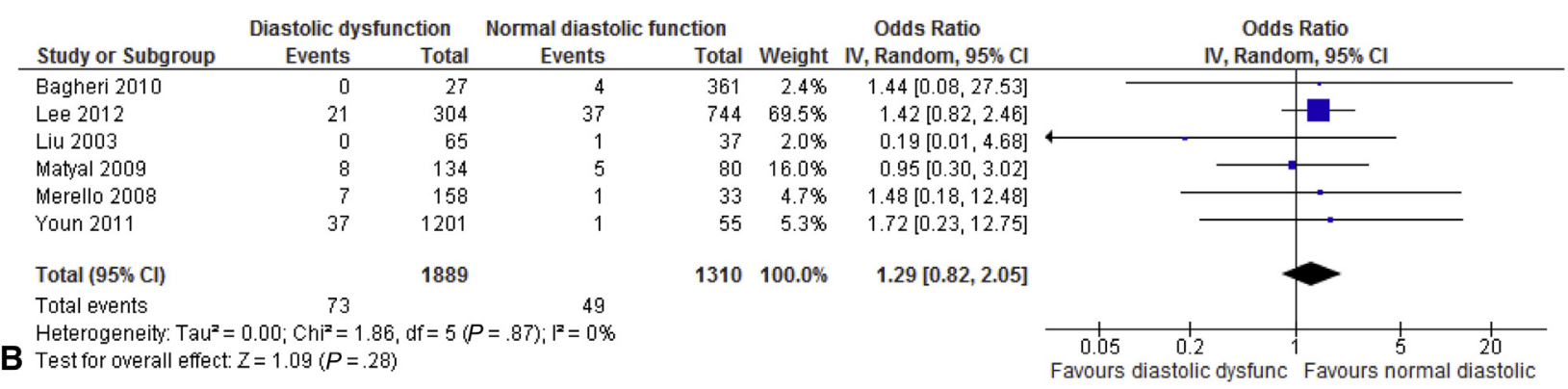

Atrial fibrillation

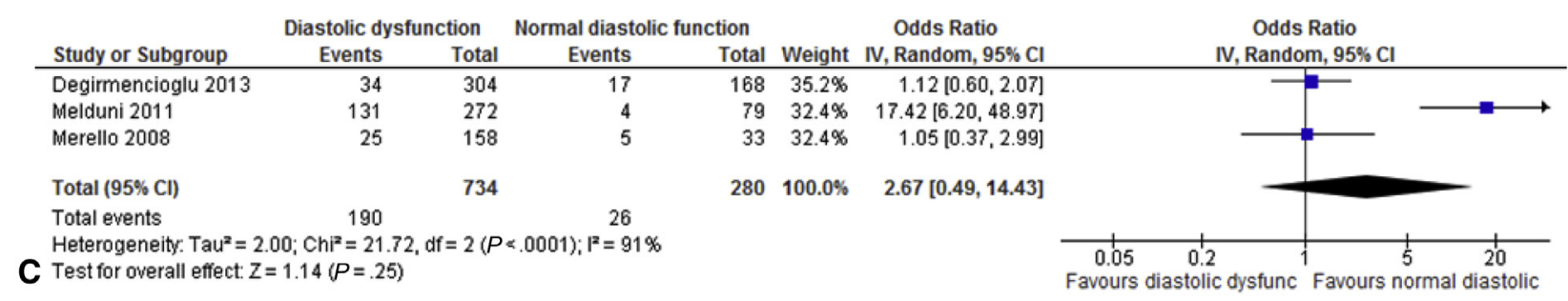

Prolonged mechanical ventilation

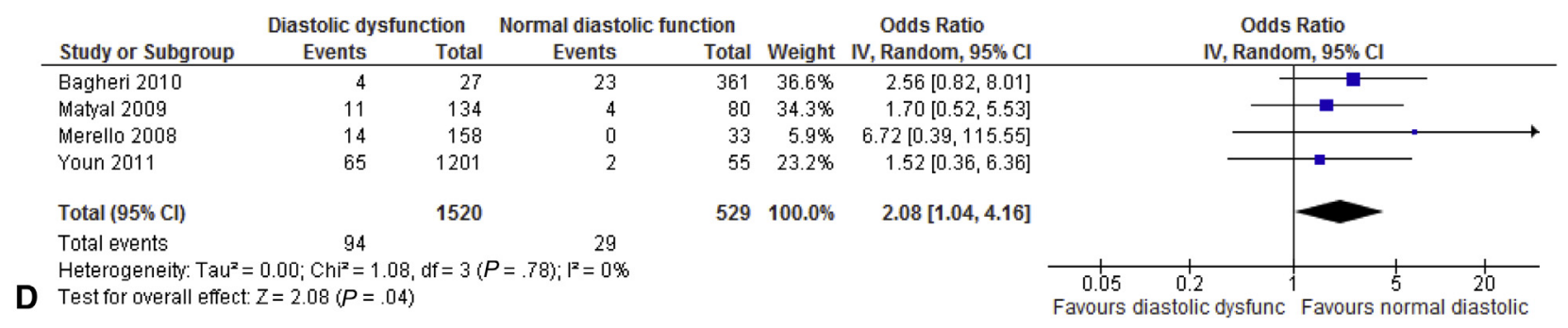

FIGURE 4. Complications after cardiovascular surgery in patients with diastolic dysfunction: A, MACE; B, myocardial infarction; C, atrial fibrillation; D, prolonged mechanical ventilation. $M A C E$, Major adverse cardiovascular events; $I V$, inverse variance; $C I$, confidence interval.

least $20 \%$ of patients undergoing cardiac surgery in whom diastolic dysfunction cannot be accurately measured. ${ }^{34}$ Adjusted risk estimates wherever available are provided in Table E1. As far the confounding from diastolic dysfunction associated with possible ischemic heart disease, our subgroup analysis found no impact of LV systolic dysfunction on the relationship between diastolic dysfunction and mortality. Individual studies also have shown that LVEF and left main disease did not provide independent prognostic value over LV end-diastolic diameter and deceleration time in predicting mortality. ${ }^{23,24}$ We were limited in the use of studies that provided E/e as the measure of diastolic dysfunction ${ }^{35}$ that is known to limit confounding from moderate-to-severe aortic stenosis and secondary mitral regurgitation. ${ }^{36,37}$ Also, different measures of diastolic dysfunction were used among studies; however, grading of diastolic dysfunction was reported consistently across studies. As far the impact of diastolic dysfunction on vascular surgery, we could only find one study. Scarcity of outcomes and studies 
complicated the analyses; however, we used the MantelHaenszel fixed-effects model to provide the best effect estimates in this situation and sensitivity analyses with the arcsine method (random effects model) provided similar results. The use of random effects models usually is the best choice when authors believe that there is substantial methodologic heterogeneity (differences in populations, sample sizes, interventions, controls, follow up times, outcomes) or statistical heterogeneity (differences in effects) across studies; however, the use of fixed effects is the primary choice when the number of outcomes is scarce (ie, $<10 \%$ of the group total) or when there is imbalance of groups (different sample sizes) in most of the studies. In these cases, the fixed effects models give a more robust effect metric in comparison to the random effects model. As sensitivity analysis random effects model should also be used to evaluate the consistency or not of the effects between the two models. Additionally, this work is meant to be hypothesis generating and would need to be confirmed in future prospective studies.

In summary, the true and independent impact of diastolic dysfunction of postoperative cardiovascular outcomes is difficult to assess. The current risk scoring systems do not include diastolic dysfunction for risk stratification and may need to be re-evaluated.

\section{Conflict of Interest Statement}

Dr Deepak L. Bhatt discloses the following relationshipsAdvisory Board: Cardax, Elsevier Practice Update Cardiology, Medscape Cardiology, Regado Biosciences; Board of Directors: Boston VA Research Institute, Society of Cardiovascular Patient Care; Chair: American Heart Association Quality Oversight Committee; Data Monitoring Committees: Duke Clinical Research Institute, Harvard Clinical Research Institute, Mayo Clinic, Population Health Research Institute; Honoraria: American College of Cardiology (Senior Associate Editor, Clinical Trials and News, ACC.org), Belvoir Publications (Editor in Chief, Harvard Heart Letter), Duke Clinical Research Institute (clinical trial steering committees), Harvard Clinical Research Institute (clinical trial steering committee), HMP Communications (Editor in Chief, Journal of Invasive Cardiology), Journal of the American College of Cardiology (Guest Editor; Associate Editor), Population Health Research Institute (clinical trial steering committee), Slack Publications (Chief Medical Editor, Cardiology Today's Intervention), Society of Cardiovascular Patient Care (Secretary/Treasurer), WebMD (CME steering committees); Other: Clinical Cardiology (Deputy Editor), NCDRACTION Registry Steering Committee (Vice-Chair), VA CART Research and Publications Committee (Chair); Research Funding: Amarin, AstraZeneca, Bristol-Myers Squibb, Eisai, Ethicon, Forest Laboratories, Ischemix,
Medtronic, Pfizer, Roche, Sanofi Aventis, The Medicines Company; Royalties: Elsevier (Editor, Cardiovascular Intervention: A Companion to Braunwald's Heart Disease); Site Co-Investigator: Biotronik, Boston Scientific, St. Jude Medical; Trustee: American College of Cardiology; Unfunded Research: FlowCo, PLx Pharma, Takeda. Dr Bueno reports having received consulting/speaking fees from Abbott, Astra-Zeneca, Bayer, BMS-Pfizer, DaichiiSankyo, Eli-Lilly, Ferrer, Menarini, Novartis, Sanofi, Servier, and a research grant from Astra-Zeneca. All other authors have nothing to disclose with regard to commercial support.

\section{References}

1. Shahian DM, O'Brien SM, Filardo G, Ferraris VA, Haan CK, Rich JB, et al; Society of Thoracic Surgeons Quality Measurement Task Force. The Society of Thoracic Surgeons 2008 cardiac surgery risk models: part 1 - coronary artery bypass grafting surgery. Ann Thorac Surg. 2009;88(1 Suppl):S2-22.

2. Nashef SA, Roques F, Michel P, Gauducheau E, Lemeshow S, Salamon R European system for cardiac operative risk evaluation (EuroSCORE). Eur J Cardiothorac Surg. 1999;16:9-13.

3. Hannan EL, Kilburn H Jr, Racz M, Shields E, Chassin MR. Improving the outcomes of coronary artery bypass surgery in New York State. JAMA. 1994;271: 761-6.

4. Parsonnet V, Dean D, Bernstein AD. A method of uniform stratification of risk for evaluating the results of surgery in acquired adult heart disease. Circulation. 1989;79(6 Pt 2):I3-12.

5. Higgins TL, Estafanous FG, Loop FD, Beck GJ, Blum JM, Paranandi L. Stratification of morbidity and mortality outcome by preoperative risk factors in coronary artery bypass patients. A clinical severity score. JAMA. 1992;267: 2344-8.

6. Moher D, Liberati A, Tetzlaff J, Altman DG, PRISMA Group. Preferred reporting items for systematic reviews and meta-analyses: the PRISMA statement. Ann Intern Med. 2009;151:264-9. W264.

7. Hernandez AV, Walker E, Ioannidis JPA, Kattan MW. Challenges in meta-analysis of randomized controlled trials for rare harmful cardiovascular events: the case of rosiglitazone. Am Heart J. 2008;156:23-30.

8. Shuster JJ. Empirical vs natural weighting in random effects meta-analysis. Stat Med. 2010;29:1259-65.

9. IntHout J, Ioannidis JP, Borm GF. The Hartung-Knapp-Sidik-Jonkman method for random effects meta-analysis is straightforward and considerably outperforms the standard DerSimonian-Laird method. BMC Med Res Methodol. 2014; $14: 25$.

10. Viechtbauer W. Conducting meta-analyses in $\mathrm{R}$ with the metafor package. J Stat Software. 2010;36:1-48.

11. Stang A. Critical evaluation of the Newcastle-Ottawa scale for the assessment of the quality of nonrandomized studies in meta-analyses. Eur J Epidemiol. 2010; 25:603-5.

12. Denault AY, Couture P, Buithieu J, Haddad F, Carrier M, Babin D, et al. Left and right ventricular diastolic dysfunction as predictors of difficult separation from cardiopulmonary bypass. Can J Anaesth. 2006;53:1020-9.

13. Değirmencioğlu A, Şenay Ş, Güllü Ü, Zencirci E, Karakuş G, Uğur M, et al. The effect of mild left ventricular diastolic dysfunction on outcome after isolated coronary bypass surgery. Kardiol Pol. 2014;72:541-5.

14. Sastry P, Theologou T, Field M, Shaw M, Pullan DM, Fabri BM. Predictive accuracy of EuroSCORE: is end-diastolic dysfunction a missing variable? Eur J Cardiothorac Surg. 2010;37:261-6.

15. Matyal R, Hess PE, Subramaniam B, Mitchell J, Panzica PJ, Pomposelli F, et al. Perioperative diastolic dysfunction during vascular surgery and its association with postoperative outcome. J Vasc Surg. 2009;50:70-6.

16. Salem R, Denault AY, Couture P, Belisle S, Fortier A, Guertin MC, et al. Left ventricular end-diastolic pressure is a predictor of mortality in cardiac surgery independently of left ventricular ejection fraction. Br J Anaesth. 2006;97: $292-7$. 
17. Youn YN, Shim CY, Yang H, Hong S, Chung N, Yoo KJ. Effect of diastolic dysfunction on early outcomes during elective off-pump coronary artery bypass grafting: a prospective observational study. Ann Thorac Surg. 2011; 92:587-93.

18. Lee EH, Yun SC, Chin JH, Choi DK, Son HJ, Kim WC, et al. Prognostic implications of preoperative E/e' ratio in patients with off-pump coronary artery surgery. Anesthesiology. 2012;116:362-71.

19. Bagheri J, Rezakhanloo F. Dose impaired relaxation of left ventricle affect early outcomes in CABG patients? Acta Med Iran. 2010;48:164-7.

20. Merello L, Riesle E, Alburquerque J, Torres H, Aranguiz-Santander E, Pedemonte O, et al. Risk scores do not predict high mortality after coronary artery bypass surgery in the presence of diastolic dysfunction. Ann Thorac Surg. 2008;85:1247-55.

21. Afilalo J, Flynn AW, Shimony A, Rudski LG, Agnihotri AK, Morin JF, et al. Incremental value of the preoperative echocardiogram to predict mortality and major morbidity in coronary artery bypass surgery. Circulation. 2013; 127:356-64.

22. Melduni RM, Suri RM, Seward JB, Bailey KR, Ammash NM, Oh JK, et al. Diastolic dysfunction in patients undergoing cardiac surgery: a pathophysiological mechanism underlying the initiation of new-onset post-operative atrial fibrillation. J Am Coll Cardiol. 2011;58:953-61.

23. Liu J, Tanaka N, Murata K, Ueda K, Wada Y, Oyama R, et al. Prognostic value of pseudonormal and restrictive filling patterns on left ventricular remodeling and cardiac events after coronary artery bypass grafting. Am J Cardiol. 2003;91: $550-4$.

24. Bernard F, Denault A, Babin D, Goyer C, Couture P, Couturier A, et al. Diastolic dysfunction is predictive of difficult weaning from cardiopulmonary bypass. Anesth Analg. 2001;92:291-8.

25. Lawrie GM, Morris GC Jr. Factors influencing late survival after coronary bypass surgery. Ann Surg. 1978;187:665-76.

26. Lappas DG, Skubas NJ, Lappas GD, Ruocco E, Tambassis E, Pasque M. Prevalence of left ventricular diastolic filling abnormalities in adult cardiac surgical patients: an intraoperative echocardiographic study. Semin Thorac Cardiovasc Surg. 1999;11:125-33.

27. Djaiani GN, McCreath BJ, Ti LK, Mackensen BG, Podgoreanu M, PhillipsBute B, et al. Mitral flow propagation velocity identifies patients with abnormal diastolic function during coronary artery bypass graft surgery. Anesth Analg. 2002;95:524-30. table of contents.
28. Shi Y, Denault AY, Couture P, Butnaru A, Carrier M, Tardif JC. Biventricular diastolic filling patterns after coronary artery bypass graft surgery. $J$ Thorac Cardiovasc Surg. 2006;131:1080-6.

29. Vaskelyte J, Stoskute N, Kinduris S, Ereminiene E. Coronary artery bypass grafting in patients with severe left ventricular dysfunction: predictive significance of left ventricular diastolic filling pattern. Eur $J$ Echocardiogr. 2001;2:62-7.

30. Higashita R, Sugawara M, Kondoh Y, Kawai Y, Mitsui K, Ohki S, et al. Changes in diastolic regional stiffness of the left ventricle before and after coronary artery bypass grafting. Heart Vessels. 1996;11:145-51.

31. Oppizzi M, Zoia E, Franco A, Gerli C, Vendrame G, Guarracino F, et al Diastolic dysfunction in cardiac surgery intensive care. Study methods, changes and prognosis [in Italian]. Minerva Anestesiol. 1997;63:29-38.

32. Lawson WE, Seifert F, Anagnostopoulos C, Hills DJ, Swinford RD, Cohn PF Effect of coronary artery bypass grafting on left ventricular diastolic function. Am J Cardiol. 1988;61:283-7.

33. Bhatti F, Grayson AD, Grotte G, Fabri BM, Au J, Jones M, et al; North Wes Quality Improvement Programme in Cardiac Interventions. The logistic EuroSCORE in cardiac surgery: how well does it predict operative risk? Heart. 2006;92:1817-20.

34. Apostolakis EE, Baikoussis NG, Parissis H, Siminelakis SN, Papadopoulos GS Left ventricular diastolic dysfunction of the cardiac surgery patient; a point of view for the cardiac surgeon and cardio-anesthesiologist. J Cardiothorac Surg. 2009;4:67.

35. Jun NH, Shim JK, Kim JC, Kwak YL. Prognostic value of a tissue Doppler-derived index of left ventricular filling pressure on composite morbidity after off-pump coronary artery bypass surgery. Br J Anaesth. 2011;107:519-24.

36. Bruch C, Stypmann J, Gradaus R, Breithardt G, Wichter T. Usefulness of tissue Doppler imaging for estimation of filling pressures in patients with primary or secondary pure mitral regurgitation. Am J Cardiol. 2004;93:324-8.

37. Bruch C, Stypmann J, Grude M, Gradaus R, Breithardt G, Wichter T. Tissue Doppler imaging in patients with moderate to severe aortic valve stenosis: clinical usefulness and diagnostic accuracy. Am Heart J. 2004; 148:696-702.

Key Words: diastolic dysfunction, cardiovascular surgical procedures, mortality, meta-analysis 